\title{
ANOXEMIA IN PNEUMONIA AND ITS RELIEF BY OXYGEN INHALATION
}

\author{
BY CARL A. L. BINGER \\ (From the Hospital of The Rockefeller Institute for Medical Research, New York)
}

(Received for publication July 30, 1928)

\section{INTRODUCTION}

Among those who have had most experience with the therapeutic use of oxygen in pneumonia, there seems at present to be fairly general agreement as to its value (1) (2) (3) (4) (5). The situation may perhaps be summed up this way: Oxygen in pneumonia is useful in so far as it can relieve or abolish anoxemia. It has no specific effect on the invading microörganism. It does, however, tend to restore to its normal state the internal environment of the host. This must be looked upon as an advantage in the patient's struggle for survival. There is ample evidence for the fact that acute anoxemia is harmful (6).

When used early and continuously, and in the proper concentration, oxygen inhalation probably prolongs the patient's life. How often it actually is responsible for saving life it is impossible to state. Both the complexity and paucity of the material make statistical proof not only difficult to marshal, but of questionable value. An occasional brilliant recovery in a patient desperately ill with pneumonia, who has received oxygen treatment, seems a cogent argument in favor of the value of this form of therapy. But post hoc reasoning is not impregnable, and brilliant recoveries occur without oxygen. It may be wisest to be skeptical as to the life saving value of oxygen in pneumonia, but there can be no doubt that its use is indicated in the presence of anoxemia.

Fortunately, we have a criterion for the efficaciousness of oxygen inhalation other than the survival of the patient. We refer to the effect on cyanosis, more particularly its effect on the oxygen content and per cent saturation of the arterial blood. The method of arterial 
puncture introduced by Hürter (7) and Stadie (8) has been of the greatest service in putting this subject on a sound basis. For routine clinical use, to be sure, such analyses are perhaps not necessary; the presence and disappearance of cyanosis being a fairly adequate guide. But in attempting to discover the causes of anoxemia and to evaluate the proper place of oxygen inhalation as a therapeutic procedure, we should still be at sea without this test.

In spite of the wide use of oxygen in pneumonia, comparatively few figures have been published concerning the degree of arterial oxygen unsaturation which occurs in the disease or the changes in saturation which follow oxygen therapy. Our study presents the results of over 300 arterial punctures and oxygen analyses made in 137 patients. The paper is divided into two parts, the first dealing with frequency tables having to do with the distribution of arterial oxygen saturations and a consideration of them in relation to the type of invading organism, survival of patients, and the effect of oxygen inhalation. The second part of the paper deals with a description of a few individual cases selected because of their extreme anoxemia and because in several of them autopsies were performed and long findings observed not long after the oxygen analyses of the arterial blood were performed.

\section{MATERIAL AND METHODS}

The patients studied were all admitted to the Hospital of The Rockefeller Institute with the diagnosis of acute pneumonia. The great majority were pneumonias of the lobar type, though in a few the distribution of the signs and the clinical history were that of bronchopneumonia. No selection was made in this series with the exception that in general arterial bleedings were performed on the more seriously ill and on those with the more intense cyanosis.

The blood was usually obtained from the femoral artery with the technique suggested by Fraser (9). No harm or inconvenience was ever found to come from this procedure in approximately 300 punctures. One patient was bled nine times in 12 days without ill effect. The blood was transferred from the syringe to small tonometers, where it was oxalated and kept over mercury. In most instances duplicate analyses of oxygen content and capacity were 
made directly after bleeding. The analytical method of Van Slyke and Neill (10) was used.

Oxygen was administered by placing the patients in a chamber (11) arranged for the maintenance of high partial pressures of oxygen with adequate provision for the removal of $\mathrm{CO}_{2}$, heat and moisture. In most instances a concentration of 40 per cent was used. This has been found empirically to be sufficient to restore the oxygen in the blood to a normal level, when it can be restored. Sometimes concentrations of 50 per cent were used. In the light of the toxic effects of high partial pressures of oxygen (12) (13) it does not seem wise to expose patients to concentrations higher than 60 per cent.

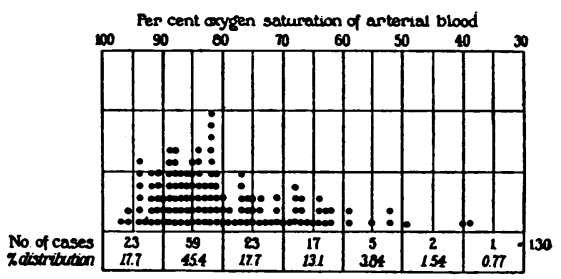

Fig. 1. Frequency Table Showing Distribution of Per Cent Oxygen Saturation of Arterial Blood in 130 Cases of Acute Pneumonia

PART I

The per cent saturation of the arterial blood in 130 patients with pneumonia has been tabulated on a frequency chart (fig. 1). The patients were all bled during the acute stage of the disease while breathing room air. Nearly 50 per cent of the analyses fell in a zone between 80 and 89 per cent saturation. With the exception of 8 patients whose oxygen saturation was below 60 per cent, the remainder of the series was fairly equally distributed between 60 and 69 per cent, 70 and 79 per cent and 90 and 97 per cent. Inspection of the chart will make this clear.

When tabulated according to the type of invading microörganism, the distribution will be found to be fairly similar for all types (fig. 2). In each instance the greatest massing of cases occurs between saturations of 80 and 89 per cent. The fact that the distribution is more 
uniform and that there are relatively more instances of low saturation in Type III cases may possibly be related to the character of the lesion produced by this organism in which much moisture collects in the lung, but it is more probably a chance distribution due to the small number of representatives of this group which the series affords.

Stadie (8) observed in a series of 33 pneumonia cases that there was only one case which recovered in which the arterial unsaturation of the blood was greater than 20 per cent. It would be of interest to know whether in a larger series the degree of anoxemia and mortality showed as close a correlation even though they may not be caus-

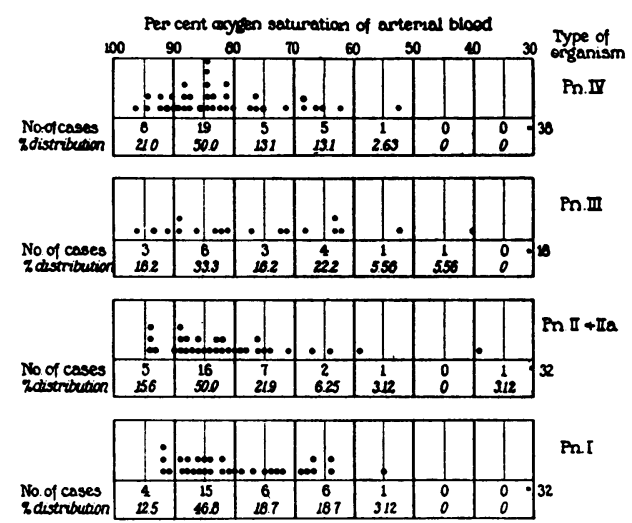

Fig. 2. Frequency Tabie Showing Distribution of Per Cent Oxygen Saturation of Arterial Blood Arranged According to THE TyPe OF INFECTING ORGANISM

ally related. Stadie's patients suffered from pneumonia following influenza in which high death rate and pronounced anoxemia were striking features. From the present series no statistical data of exactly this nature are available, since many of our patients were treated in the oxygen chamber. One can, however, consider mortality in relation to the per cent saturation of the arterial blood during the acute stage of the disease, irrespective of any therapeusis such as serum administration or oxygen. A frequency table of this sort has been constructed (fig. 3). It appears that of the surviving patients only 21 out of 83 , or approximately 25 per cent, had a saturation under 
80 per cent, while of the fatal cases there were 27 out of 47 , or approximately 57 per cent. We may conclude, therefore, that in this series, too, a positive correlation exists between anoxemia and mortality since the cases which terminated fatally showed on the whole the greater degree of anoxemia. If the patients who received specific treatment with Type I antipneumococcus serum are omitted from the series, the number of surviving cases whose saturation was originally under 80 per cent becomes correspondingly smaller. This reflects the favorable influence of the serum on the more seriously ill and anoxemic. Twelve out of 58 surviving patients untreated by serum, or 20.7 per cent, showed a saturation of under 80 per cent, whereas 21 out of 37 , or 57.8 per cent of the fatal cases untreated by serum had

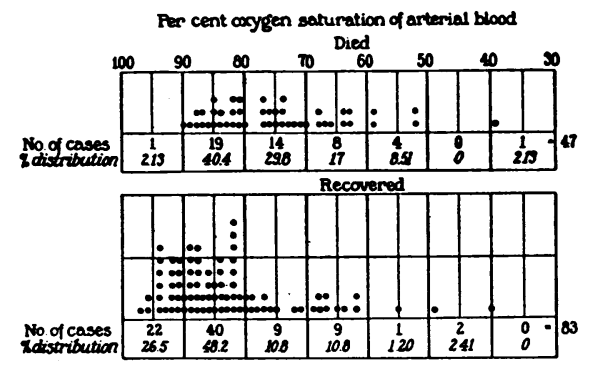

Fig. 3. Frequency Table Showing Comparison of Distribution of Arterial Oxygen Saturation in Fatal and Recovered Cases

blood circulating in their arteries which was less than 80 per cent saturated. For the details of this group figure 4 should be consulted.

These facts, though less convincing than they would be if none of the patients had been given oxygen, still show a definite correlation between anoxemia and mortality. It was, of course, out of the question to withhold oxygen for the purpose of compiling statistics. Something, however, can be learned from a consideration of the per cent saturation of the arterial blood after the patient has been breathing 40 per cent oxygen in the chamber for several hours. The character of the response appears to be of prognostic significance. Of 51 patients, treated in the chamber, who recovered from pneumonia, 41 , or 80 per cent, showed a saturation of their arterial blood above 90 per cent, while only 19 per cent ( 7 of 36 ) of the fatal chamber cases 
reached this level (fig. 5). The reasons why the more gravely ill patients fail to reach a normal arterial oxygen saturation when exposed to 40 per cent oxygen will be discussed in the consideration of individual patients in the second part of this paper.

In spite of the fact that fatal cases apparently less often reach a saturation of 90 per cent in the chamber than those which survive,

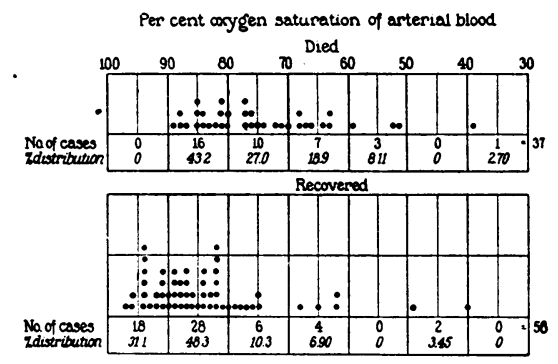

Fig. 4. Frequency Table Showing Comparison of Distribution of Arterial Oxygen Saturation in Fatal and Recovered Cases Not Treated by Type I Antipneumococcus Serum

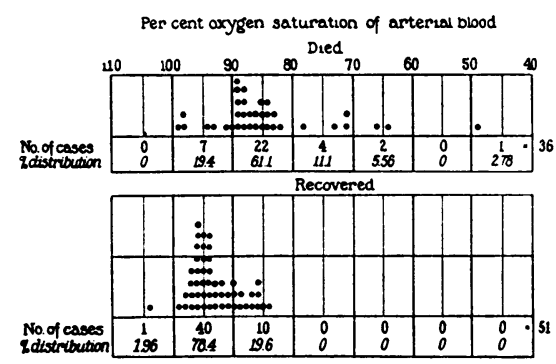

Fig. 5. Frequency Table Showing Distribution of Arterial Oxygen Saturation in Patients after Exposure to $40 \pm$ Per Cent Oxygen. Comparison of Fatal and Recovered Cases

the per cent increase in arterial saturation of the two groups when tabulated on a frequency chart shows a similar distribution for both (fig. 6). The figure charted is the difference between the per cent. saturation before and after admission to the chamber, expressed in terms of per cent of the value before admission. Attention should be called to the fact that only one case of 48 surviving (approximately 
2 per cent) showed an actual drop in per cent saturation after being placed in the chamber, while 3 of 36 fatal cases (approximately 8 per cent) behaved in this manner. With this exception, however, no well defined difference exists between the two groups.

Since the fatal cases show an increase in per cent saturation quite comparable to those surviving but do not so frequently reach a level of 90 per cent, and since they are in general more anoxemic to begin with than the ones which recover, the implication seems justifiable that a higher partial pressure of oxygen in the chamber might have restored the more seriously sick as well to the normal level. Concentrations higher than 50 per cent have not been used, partly because they are

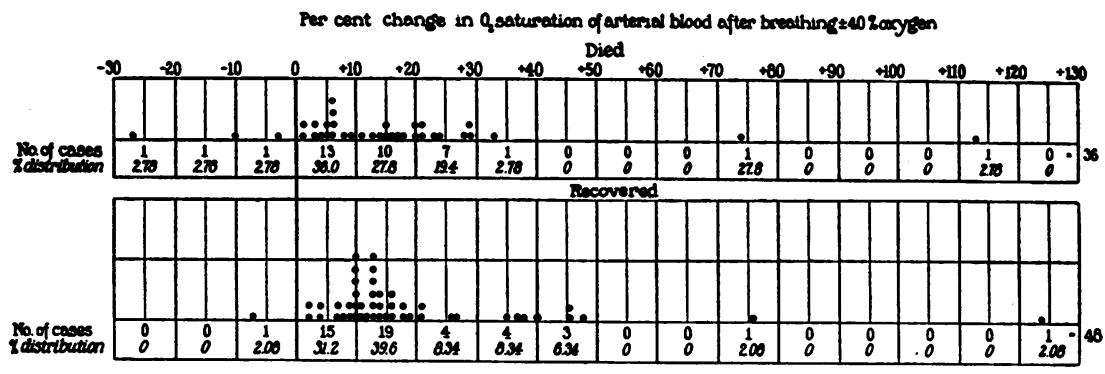

Fig. 6. Frequency Table Showing Per Cent Change in Oxygen Saturation of Arterial Blood After the Patients Had Inhaled $40 \pm$ Per Cent Oxygen for Several Hours. Comparison of Fatal and Recovered Cases

difficult and expensive to maintain when there is frequent ingress and egress from the chamber as there must be in caring for a sick patient, partly because it does not seem wise to approach too closely the toxic concentration of oxygen in the presence of an already damaged lung and elevated temperature (14).

The division of these cases into two groups, one which recovered and one which succumbed, is perhaps not a logical one, but it is convenient. Death and recovery in pneumonia in the absence of specific therapy must still be looked upon as accidental. It seems safe to assume that the fatal cases were on the whole more seriously ill throughout than the others, and from this point of view the classification is useful. 


\section{PART II}

Eight of 130 cases tabulated in figure 1 showed an oxygen saturation below 60 per cent. A more detailed consideration of these cases will be profitable both from the point of view of inquiring into the causes of anoxemia and of explaining why certain patients, when placed in the chamber, exhibit no increase in the per cent oxygen saturation of their arterial blood.

Case 1. (Hospital No. 5811.) Lobar pneumonia, septicemia (Pneumococcus Type II). Died.

The patient was a male, aged 37, who entered the hospital after 2 days of acute illness. On admission there was slight cyanosis and evidence of pneumonic consolidation of the upper two-thirds of the right lung. Three days later the patient's condition became definitely worse. Cyanosis was intense and diffuse and the patient could be aroused only with difficulty. The signs in the right chest had extended to the base, where many medium and coarse râles could be heard.

The arterial blood was only 39.4 per cent saturated with oxygen, the content and capacity being 5.94 and 15.06 vols. per cent respectively. At this time the patient was breathing 30 to the minute. He was transferred to the oxygen chamber and kept in an atmosphere containing 42 per cent oxygen until he died, approximately 16 hours later. One and one-half hours after admission to the chamber the arterial $\mathrm{O}_{2}$ content was 14 vols. per cent, the capacity 16.7 vols. per cent and the per cent saturation 83.8. The respiratory rate at this time was 36. Twelve hours before death blood culture showed 29 colonies of Type II pneumococcus per cubic centimeter of blood. No permission for autopsy was granted.

\section{Comment}

The significant feature of this case is the intense anoxemia coming on simultaneously with a spread to the right lower lobe. The fact that in this region there was only moderate dulness but many medium and coarse râles appears to be of importance. It is this type of freshly spread early lesion in the presence of a heavy blood invasion which is so frequently associated with intense arterial anoxemia.

Case 2. (Hospital No. 5418.) Lobar pneumonia (Pneumococcus Type II). Died.

The patient, a male, aged 56, entered the hospital on the second day of illness. There was evidence of pneumonic consolidation in the right lower lobe, with coarse râles and a friction rub to be heard in the left scapular region. The patient's condition gradually became worse. Four days later both lungs were full 
of coarse râles which masked the other signs except for dulness and bronchial breathing in the upper two-thirds of the left lung and the lower half of the right lung. An arterial puncture was done while the patient was breathing room air. The blood was only 59.4 per cent saturated, having an oxygen content and capacity of 6.25 , and 10.5 vols. per cent respectively. The patient was transferred to the oxygen chamber, but died within 12 hours, before a second arterial puncture was performed. Autopsy showed the right lung to be in the early stage of red hepatization, weighing 800 grams. The left lung weighed 1320 grams, the upper lobe being completely solidified and in the stage of gray hepatization. The lower lobe was similar in consistency and appearance to the right lung.

\section{Comment}

During life the patient showed an extensive pulmonary involvement with physical signs characterized by loud, diffuse râles. Autopsy showed heavy red lungs. It is this combination of early, rapidly spreading pneumonia, with much moisture, in which anoxemia is most intense.

Case 3. (Hospital No. 5217.) Bronchopneumonia (Streptococcus hrmolyticus). Died.

The patient, a male, aged 31, was admitted after 3 days of acute illness. There was intense cyanosis of the face and nail beds. Chest signs indicated involvement of the left lower lobe, where there was dulness with many medium, moist râles posteriorly. There was also evidence of fluid in the right chest. A hemolytic streptococcus was recovered from the patient's blood stream.

The arterial blood was 58.8 per cent saturated. The patient was transferred to the oxygen chamber. No improvement in color was apparent. A second arterial puncture was not done. The next morning $300 \mathrm{cc}$. of yellow, cloudy fluid were withdrawn from the right thoracic cavity. An hour later the patient died, 13 hours after admission.

Autopsy showed the right lung to be non-air-containing and of a leathery consistency. It weighed 610 grams, and was dark red to purple in color. Numerous small abscesses were found throughout the lower lobe. The left lung weighed 400 grams and contained air. No areas of consolidation were present.

\section{Comment}

Oxygen want may perhaps be explained in this patient by a shunt of blood through the right lung, which was found to be non-air-containing at autopsy. This will also explain why there was no improvement in the patient's color. 
Case 4. (Hospital No. 5413.) Male, aged 52. Lobar pneumonia (Pneumococcus Type III). Died.

The patient came to the hospital on the third day of illness. There was marked diffuse cyanosis. The arterial blood was 52.1 per cent saturated. Signs of consolidation were confined to the lower right back, where there were numerous moist râles. Musical râles were scattered throughout the chest on both sides and expiration was prolonged.

The patient was transferred to the oxygen chamber, which was charged to 41.5 per cent $\mathrm{O}_{2}$. Twelve hours later dyspnea and cyanosis were unimproved. Breathing was asthmatic. Breath sounds were suppressed over the lower half of the right chest in back, where there were dulness and fine râles. There was much mucus in the upper respiratory tract. The $\mathrm{O}_{2}$ content was now 12.45 and capacity 13.75 vols. per cent. The per cent saturation was therefore 90.6. The next 12 hours brought a distinct change for the worse. The patient's face was purple and he was gasping for breath. Loud tracheal râles were present. Examination of the chest was not satisfactory because of patient's condition. Blood culture showed 10 colonies of Pneumococcus Type III-per cubic centimeter of blood. The arterial blood had dropped to a saturation of 62.5 per cent, the content and capacity being 4.40 and 7.05 respectively.

At autopsy the left lung was found to be quite normal and weighed 550 grams. The right lung, however, weighed over 2 kilograms. On section all three lobes appeared to be equally consolidated. The cut surface was firm, granular, dark reddish and quite moist. Frothy, bloody fluid exuded from the smaller bronchi.

\section{Comment}

The first response to oxygen was a satisfactory one as far as the saturation of the arterial blood was concerned. With increasing amount of moisture in the air spaces of the right lung aeration of the blood was impeded and marked anoxemia occurred in spite of oxygen inhalation. The autopsy findings provided a satisfactory explanation for this, there being an involvement of the entire right lung which contained no air and much fluid.

Case 5. (Hospital No. 5490.) Female, aged 48. Lobar pneumonia (Pneumococcus Type I). Recovered.

Four days before admission the patient had a sudden shaking chill. On admission she appeared to be very ill. There was intense cyanosis of the finger tips. Signs of consolidation were present in the left lower lobe. Blood culture showed 3 colonies of Pneumococcus Type I per cubic centimeter. The following day the patient's condition was somewhat worse. Blood culture was still positive. She was very cyanotic. Temperature was $103.4^{\circ} \mathrm{F}$., pulse 130 , respirations 38 . The arterial saturation was 55.2 per cent, $\mathrm{O}_{2}$ content 6.76 and $\mathrm{O}_{2}$ capacity 12.26 vols. 
per cent. She was transferred to the oxygen chamber and exposed to 45 per cent oxygen. Antipneumococcus serum therapy was begun. Five and three-quarter hours after oxygen administration was started the per cent saturation had risen to 87.9 , the $\mathrm{O}_{2}$ content and capacity being now 10.41 and 11.85 vols. per cent respectively. The next day the blood culture became sterile and remained so. On January 4th the saturation had risen to 98.2 per cent. The general clinical picture was suggestive of empyema. $20 \mathrm{cc}$. of turbid, yellow fluid, from which Pneumococcus Type I was cultivated, were withdrawn from the left chest. The patient was removed from the chambe for an X-ray examination, which showed diffuse clouding of the whole left lung. When breathing room air cyanosis recurred slightly, and the respiratory rate rose from 34 to 40 . Arterial saturation had correspondingly fallen to 89.8 per cent. The next day nearly $300 \mathrm{cc}$. of turbid yellow fluid were aspirated from the left chest. The patient was subsequently transferred to another hospital for operation and eventually made a good recovery.

\section{Comment}

The case presents the interesting situation of an intense anoxemia at the outset of the disease, which not only yielded to oxygen therapy but subsequently did not recur in room air although extensive physical signs persisted. Two changes, however, had occurred: (1) The blood culture was no longer positive, and (2) a large amount of fluid had accumulated in the chest. It is not unlikely that a generalized pneumonitis may result from a persistent bacteriemia and that this may account for the apparent relationship between septicemia and anoxemia. That accumulation of fluid in the chest should be associated with a disappearance of anoxemia may, perhaps, be explained by a shutting off, or at least a diminution, of the circulation in the compressed lung.

Case 6. (Hospital No.6174.) Male, aged 46. Bronchopneumonia. Recovered.

The patient was admitted to the hospital on the seventh day of his illness, which began with a sudden sense of weakness and stabbing pain in the chest. On admission he looked sick and was obviously cyanosed. Examination of the chest showed no impairment of the percussion note. There were, however, plentiful coarse râles, most numerous at the left base, but also present at the right base. There was a faint but definite friction rub heard in the axilla. X-ray showed patchy opacity at the right hilum with some mottling at both bases. Blood culture was negative. The sputum showed that $B$. influenze was the predominant organism. Two days after admission cyanosis was more intense. Temperature was $100.8^{\circ} \mathrm{F}$., pulse 120 and respirations 28 . Arterial puncture showed the blood 
to be only 48.9 per cent saturate. $\mathrm{O}_{2}$ content was 6.43 vols. per cent, capacity 13.15. The patient was transferred to the oxygen chamber and exposed to 41.5 per cent $\mathrm{O}_{2}$. The next morning, 123 hours later, his temperature had dropped to $98.8^{\circ} \mathrm{F}$., pulse 88 and respirations 24 . The oxygen saturation was now 86.1 per cent, $\mathrm{O}_{2}$ content 9.62 and capacity 11.15 vols. per cent. Only a trace of cyanosis remained. Two days later the patient was removed from the chamber. He soon became slightly cyanosed about the face and nail beds and did not look nearly so well as when in the chamber. However, he made a steady recovery.

\section{Comment}

This case is typical of the influenzal bronchopneumonias in which extreme oxygen want is a prominent feature with pulmonary signs characterized by widespread coarse râles rather than evidence of localized consolidation. It is these patients for whom oxygen therapy is especially useful. The drop in temperature following admission to the chamber is, in the light of Boothby's observations (5), perhaps more than a coincidence.

Case 7. (Hospital No. 6213.) Male, aged 49. Lobar pneumonia (Pneumococcus Type III). Recovered.

The patient was taken sick 3 days before he entered the hospital. On admission on December 1, 1927, he showed only slight cyanosis of the lips and nail beds. At this time the lung signs consisted of suppression of breath sounds and friction rub in the right axilla, with dulness and almost completely suppressed breath sounds in the right back, below the angle of the scapula. Two days later cyanosis was slightly increased and the physical signs suggested a spread to the upper lobe of the right lung. Numerous dry râles were now present. X-ray showed opacity throughout the right upper lobe and parts of middle and lower lobes. The next day the patient was obviously worse. There had been a still further spread in the right axilla. Cough was very troublesome, and it was necessary to give codeine. At 9:15 p.m. he was given $12 \mathrm{mgm}$. of morphine. Not long after the patient's respiratory rate dropped to 10 , cyanosis became intense, and he developed CheyneStokes breathing. His lungs were filling with fluid and he rapidly passed into coma. An arterial puncture showed the blood to be only 40.2 per cent saturated with oxygen, the content being 6.86 vols. per cent and the capacity 17.08 .

He was hurriedly transferred to the oxygen chamber with immediately beneficial results. The cyanosis almost completely disappeared, respirations became regular and increased in rate to 28 and there was a striking improvement in the patient's psyche. Arterial blood drawn 12 hours after admission to the chamber showed an increase in saturation of 125 per cent. The oxygen content was now 10.59 vols. per cent, the capacity 11.74 vols. per cent and the per cent saturation 90.3. This was true in spite of the fact that the physical signs showed a still fur- 
ther spread and that coarse, bubbling râles were fairly widespread throughout the right chest. From this time on the patient made a gradual, steady recovery, and was discharged from the hospital on January 2, 1928.

\section{Comment}

The case presents a dramatic instance of the beneficial effect of oxygen. There is every reason to believe that the patient would have died had oxygen not been administered. The cumulative effects of opiates, added to a rapidly spreading pneumonitis, suddenly precipitated the patient into a profound state of oxygen want hardly compatible with life.

Case 8. (Hospital No. 5812.) Male, aged 62. Lobar pneumonia; septicemia (Pneumococcus Group IV). Died.

Four days before admission the patient had a chill and a temperature of $104^{\circ} \mathrm{F}$. The next day there were signs of pneumonia at the right lower lobe. On the day of admission, December 8,1926 , the patient was breathing at the rate of 40 to the minute, his face was livid and grayish, and he looked very sick. There were signs of consolidation throughout the lower right lobe and the lower portion of the upper lobe. Throughout the right back there was bronchial breathing, with moist râles at the base. Arterial blood was $\mathbf{5 1 . 5}$ per cent saturated with oxygen. The patient was transferred to the oxygen chamber. A blood agar plate made from 2 cc. of his blood was completely covered with confluent. colonies of Group IV pneumococci. The following morning the patient's condition was decidedly worse. Respirations were very rapid and shallow. The pneumonic process had spread to the left upper lobe, leaving the lower lobe as the only uninvolved portion. The patient died at 1:10 p.m. Autopsy showed that the right lung weighed 1100 grams. The upper and middle lobes were voluminous, massive and firm, grayishred and granular on section. The lower lobe was soft, dark red, juicy and aircontaining. The left lung weighed 660 grams. The left upper lobe was similar to the right upper, but of a redder color. The left lower lobe, like the right lower, gave evidence of much congestion.

\section{Comment}

The case presents another example of a fulminating pneumonia with massive blood infection and widespread pneumonic consolidation accompanied by severe anoxemia.

These 8 cases have in common severe anoxemia associated with extensive rapidly spreading pulmonary lesions which are characterized by the presence of much moisture in the lungs. 


\section{DISCUSSION}

A statistical estimate of the therapeutic value of oxygen in pneumonia would at present have little value. The number of cases is far too small for such an analysis and the character of the material too complex. We have had to content ourselves with the construction of simple frequency tables to study the per cent distribution of the various grades of anoxemia. There appears to be a positive correlation between anoxemia and mortality. Moreover, the level of oxygen saturation in the arterial blood of a patient exposed to 40 per cent oxygen is of prognostic importance, since the survivors usually reach a level above 90 per cent, while the fatal cases do so far less often.

We have made no effort to give complete clinical reports on all our cases because the space available will not permit this. Furthermore, an analysis of this sort is extremely confusing. The patient's condition alters so quickly in pneumonia that one can seldom be sure whether the changes observed after exposure to oxygen are determined by this or are part of the normal variations of the disease. In general we may state that, aside from conspicuous improvement in color, the other changes usually encountered were slight diminution in pulse and respiratory rate and improvement in the patient's mental state. These observations are in agreement with those observed by the authors already referred to. Boothby's recent finding (5) of a reduction of fever following oxygen administration is of much interest. That this has not been striking on our cases may perhaps be accounted for by the fact that they were mostly lobar pneumonias seen as a rule on the third to fifth day of disease and not post-operative bronchopneumonias placed in a chamber shortly after the diagnosis was made.

In a small group of cases selected because of severe anoxemia (oxygen saturation below 60 per cent). we have considered the physical signs and autopsy findings in relation to the arterial oxygen saturation. The presence of extensive, rapidly spreading pulmonary lesions with much moisture, exudate or transudate, appears to be common to this group. In what manner this type of lesion brings about anoxemia we are not yet certain. It is in part due to diminished lung volume, in part due to passage of blood through unaerated channels. Such a 
shunt of blood need not imply a completely consolidated lobe but may result from the collection of intraalveolar exudate. The degree of interference with oxygenation as well as the response to oxygen inhalation will depend both upon the amount and upon the character of the exudate. The thin, watery transudate of pulmonary edema which gives rise to outspoken anoxemia in room air may yield quickly to increased partial pressures of oxygen. A thicker plastic exudate, however, may form a fairly effective barrier to the diffusion of oxygen. We have recently considered from an experimental point of view the relation of the amount of exudate to the state of the pulmonary circulation (15). Landis's (16) work affords a basis for believing that a relationship also exists between anoxemia and the transudation of fluid through the pulmonary capillaries. These factors we believe to be of greater importance in the production of anoxemia than rapid and shallow breathing, although when much restricted a diminished pulmonary ventilation aggravates the already existing anoxemia (17).

Information concerning the oxygen tension in the tissues is much to be desired. As yet no practicable methods are available which make it possible to gain this, and we must be content with a study of the per cent saturation of the arterial blood. If anoxemia is a contributory cause of death in pneumonia it is so by its action on the vital centers, particularly the heart muscle and medulla. What the normal working oxygen tensions of these organs are we need to know and to what extent they are in oxygen debt during the course of the disease.

\section{SUMMARY AND CONCLUSIONS}

In Part I we have published frequency tables showing the per cent distribution of arterial oxygen saturation in relation to the type of infecting organism, survival of the patient and response to oxygen therapy. There appears to be a positive correlation between anoxemia and mortality. The level of oxygen saturation in the arterial blood of a patient after exposure to increased oxygen tension is of prognostic significance.

In Part II we have presented brief reports on physical signs and autopsy findings in 8 of the more severely anoxemic patients, those whose arterial blood was less than 60 per cent saturated. The common feature of these cases is an extensive rapidly spreading pneu- 
monic process characterized by much moisture in the lungs. This we believe to be the most important factor in the production of oxygen want.

The author expresses his gratitude to his former associates, Drs. G. R. Brow, Douglas Boyd, R. L. Moore, J. M. Faulkner, R. V. Christie and J. S. Davis, Jr., for their coöperation and assistance in this work.

\section{BIBLIOGRAPHY}

1. (a) Barach; A. L., J. Am. Med. Assn., 1927, lxxxix, 1865. Acute Disturbance of Lung Function in Pneumonia. Methods of Oxygen Treatment.

(b) Barach, A. L., Arch. Int. Med., 1926, xxxvii, 186. Methods and Results of Oxygen Treatment in Pneumonia.

2. Stadie, Wm. C., J. Exp. Med., 1922, xxxv, 337. The Treatment of Anoxemia in Pneumonia in an Oxygen Chamber.

3. Meakins and Davies, Respiratory Function in Disease, Edinburgh, 1925, 386.

4. Binger, Carl A. L., N. Y. State J. Med., 1925, xxv, 953. Therapeutic Value of Oxygen in Pneumonia.

5. Boothby, W. M., and Haines, S. F., J. Am. Med. Assn., 1928, xc, $372 . \quad$ Oxygen Therapy.

6. Haldane, J. S., Respiration, New Haven, 1922.

7. Hürter, Deutsch Arch. f. Klin. Med., 1912, cviii, 1. Untersuchungen am arteriellen menschlichen Blute.

8. Stadie, Wm. C., J. Exp. Med., 1919, xxx, 215. The Oxygen of the Arterial and Venous Blood in Pneumonia and its Relation to Cyanosis.

9. Fraser, F. R., Graham, G., and Hilton, R., J. Physiol., 1924, lviii, p. xxxiv. A Method of Obtaining $50 \mathrm{cc}$, , or More, of Human Arterial Blood.

10. Van Slyke, D. D., and Neill, J. M., J. Biol. Chem., 1924, lxi, 523. The Determination of Gases in Blood and Other Solutions by Vacuum Extraction and Manometric Measurement.

11. Binger, Carl A. L., The Modern Hospital, 1925, xxiv, 186. The Construction and Management of an Oxygen Chamber.

12. Barach, A. L., Am. Rev. Tuberc., 1926, xiii, 293. The Effects of Atmospheres Rich in Oxygen on Normal Rabbits and on Rabbits with Pulmonary Tuberculosis.

13. Binger, Carl A. L., Faulkner, James M., and Moore, Richmond L., J. Exp. Med., 1927, xlv, 849. Oxygen Poisoning in Mammals.

14. Faulkner, James M., and Binger, Carl A. L., J. Exp. Med., 1927, xlv, 865. Oxygen Poisoning in Cold-Blooded Animals. 
15. Binger, Carl A. L., Christie, R. V., and Ehrich, Wilhelm, J. Am. Med. Assn. (in press). Diathermy in Pneumonia.

16. Landis, Eugene M., Am. J. Physiol., 1928, lxxxiii, 528. Micro-injection Studies of Capillary Permeability. III. The Effect of Lack of Oxygen on the Permeability of the Capillary Wall to Fluid and to the Plasma Proteins.

17. Binger, Carl A. L., and Davis, John Staige, Jr., J. Clin. Invest., 1928, vi, 171. The Relation of Anoxemia to the Type of Breathing in Pneumonia. 